\title{
Vestibulo-Ocular Reflex Responses to a Multichannel Vestibular Prosthesis Incorporating a 3D Coordinate Transformation for Correction of Misalignment
}

\author{
Gene Y. Fridman, ${ }^{1}$ Natan S. Davidovics, ${ }^{1,2}$ Chenkai Dai, ${ }^{1}$ Americo A. Migliaccio, ${ }^{1,2}$ \\ and Charles C. Della Santina, \\ ${ }^{1}$ Vestibular NeuroEngineering Laboratory, Department of Otolaryngology - Head E Neck Surgery, Johns Hopkins School of \\ Medicine, Ross Bldg Rm 830, 720 Rutland Ave., Baltimore, 11 MD 21205, USA \\ ${ }^{2}$ Department of Biomedical Engineering, Johns Hopkins School of Medicine, Baltimore, MD 21205, USA
}

Received: 25 June 2009; Accepted: 17 January 2010; Online publication: 23 February 2010

\begin{abstract}
There is no effective treatment available for individuals unable to compensate for bilateral profound loss of vestibular sensation, which causes chronic disequilibrium and blurs vision by disrupting vestibulo-ocular reflexes that normally stabilize the eyes during head movement. Previous work suggests that a multichannel vestibular prosthesis can emulate normal semicircular canals by electrically stimulating vestibular nerve branches to encode head movements detected by mutually orthogonal gyroscopes affixed to the skull. Until now, that approach has been limited by current spread resulting in distortion of the vestibular nerve activation pattern and consequent inability to accurately encode head movements throughout the full 3-dimensional (3D) range normally transduced by the labyrinths. We report that the electrically evoked 3D angular vestibulo-ocular reflex exhibits vector superposition and linearity to a sufficient degree that a multichannel vestibular prosthesis incorporating a precompensatory 3D coordinate transformation to correct misalignment can accurately emulate semicircular canals for head rotations throughout the range of 3D axes normally transduced by a healthy labyrinth.
\end{abstract}

Keywords: electrical, stimulation, vestibular, prosthesis, VOR, gyroscopes, implant, labyrinth, vestibulo-ocular reflex

\section{INTRODUCTION}

Bilateral loss of vestibular sensation can occur as the result of ototoxic drug exposure, ischemia, infection, genetic abnormality, or trauma to the inner ears. Because input from the vestibular labyrinths normally mediates perception of movement and reflexes that stabilize posture and visual gaze, loss of this input causes chronic disequilibrium, postural instability, and visual blur due to illusory movement of the world during head motion (Grunbauer et al. 1998; Minor 1998; Gillespie and Minor 1999). A multichannel neuro-electronic prosthesis that senses rotation of the head in three dimensions and delivers corresponding stimuli to the ampullary branches of the vestibular nerve should restore sensation of head rotation, which in turn should help to stabilize gaze and at least partially restore perception of head movement. Restoring function to just one labyrinth is a reasonable goal for such a device because the adaptive capacity of vestibulocerebellar circuits is sufficient to ensure that individuals with a single, normal labyrinth (e.g., after unilateral injury or infection) typically compensate well enough to suffer little o disability in activities of daily life (Curthoys and Halmagyi 1995; Black et al. 1996).
Correspondence to: Charles C. Della Santina · Vestibular NeuroEngineering Laboratory $\cdot$ Johns Hopkins School of Medicine $\cdot$ Ross Bldg Rm 830, 720 Rutland Ave., Baltimore, MD 21205, USA. Telephone: +1-410-5027909; fax: +1-410-6148610; email: charley.dellasantina@jhu.edu 
Normally, the eyes rotate opposite the direction of head rotation in order to stabilize images on the retinae. This compensatory eye rotation is driven by the angular vestibulo-ocular reflex (aVOR), for which sensory input is provided by three mutually orthogonal semicircular canals (SCC) in each inner ear's vestibular labyrinth. Discharge rates of vestibular afferent fibers in each SCC's ampullary nerve are modulated by the component of head angular velocity about that SCC's axis (Ewald 1892; Wilson and Melvill 1979; Carey and Della Santina 2005; Della Santina et al. 2005b). The resulting eye movements can be measured precisely in $3 \mathrm{D}$, giving insight into relative excitation of the ampullary nerves driving the reflex (Robinson and Murphy 1932; Robinson 1963; Aw et al. 1996a, b; Migliaccio et al. 2005).

Classic experiments by Cohen, Suzuki, et al. (Cohen et al. 1963, 1964; Cohen and Suzuki 1963a, b; Suzuki et al. 1964, 1969; and Suzuki and Cohen 1964) demonstrated that pulses of electrical stimulation delivered to the ampullary nerves via wire electrodes elicit an aVOR about axes of rotation similar to those elicited by the hydrodynamic excitation of the individual canals (Ewald 1892). Merfeld, Gong, Lewis, et al. (Gong and Merfeld 2000, 2002; Lewis et al. 2001, 2002; Merfeld et al. 2006, 2007; and Gong et al. 2008) described a single-channel prosthesis based on the idea of sensing rotational motion using a gyroscope and stimulating the appropriate branch of the vestibular nerve. Della Santina et al. (Della Santina et al. 2005b, 2006, 2007; Chiang et al. 2009; and Davidovics et al. 2009) extended that approach to $3 \mathrm{D}$ using a multichannel prosthesis that contains three mutually orthogonal gyroscopes to sense head rotational velocity and deliver electrical stimulus via multiple electrodes to the appropriate nerves. Tests of this device in chinchillas rendered bilaterally vestibular-deficient via gentamicin treatment revealed partial restoration of the $3 \mathrm{D}$ aVOR. However, current spread within implanted labyrinths limited the ability to selectively stimulate each ampullary nerve without incurring spurious excitation of adjacent branches of the vestibular nerve. Increasing stimulus current amplitude initially increased aVOR magnitude without changing the aVOR axis, but at higher currents, the eye rotation axis deviated from the ideal as spurious stimulation of other vestibular nerve branches altered the head rotation axis "perceived" by brainstem neurons mediating the aVOR.

In theory, one should be able to overcome the effects of current spread from an electrode targeting one ampullary nerve by adjusting the input delivered via other electrodes targeting two other ampullary nerves in the same (or contralateral) labyrinth. For example, if current intended for the horizontal ampullary nerve spuriously excites the anterior and posterior ampullary nerves, then head rotation purely about the horizontal SCC axis might be encoded by modulating not only the horizontal electrode input but instead by simultaneously modulating stimuli on all three electrodes to represent a horizontal head rotation via vector summation. If linearity and vector superposition hold, this procedure amounts to a simple linear transformation between two different 3D coordinate systems, analogous to the matrix algebra transformations popularized by Robinson (1982) for analysis of the 3D aVOR. One should be able to characterize this transformation by delivering a set of stimuli $\vec{s}_{i}$ (with each $\vec{s}_{i}$ being a 3-vector representing a triplet of stimulus intensities delivered via three electrodes targeting the three ampullary nerves) and measuring the corresponding responses $\vec{r}_{i}$ (with each $\vec{r}_{i}$ being a 3-vector representing the axis and speed of observed eye movement responses) for a set of $N$ virtual head movements spanning the range of head movement axes and rotational velocities normally encoded by the labyrinth. A single $3 \times 3$ matrix $\boldsymbol{M}$ can then be found using least-squares techniques such that

$$
\vec{r}_{i} \approx M \vec{s}_{i} \text { for } i=1 \ldots N .
$$

Once $\boldsymbol{M}$ has been established, the appropriate pattern of electrode activation $\vec{a}$ the prosthesis should deliver to the three ampullary nerve electrodes during a head rotation eliciting gyroscope signals $\vec{g}$ is

$$
\vec{a}=M^{-1} \vec{g}
$$

This procedure would require that $\boldsymbol{M}$ is computed during an occasional "fitting" session, analogous to fitting required for patients with cochlear implants. For this procedure to work properly, the axes of eye movement responses to individual stimulation of each the three ampullary nerves must be linearly independent with respect to each other. Otherwise, the inverse of $\boldsymbol{M}$ cannot exist and the matrix pseudoinverse calculation will fail. In this context, linear independence means that the 3D axis of aVOR responses to stimuli delivered via any one electrode alone cannot be in the plane defined by the axes of responses to the other two electrodes. The accuracy with which linear precompensation can correct for current spread also depends on the extent to which the electrically evoked aVOR response is linear and exhibits vector summation.

Observations by Cohen et al. (1964) during electrical stimulation of ampullary nerves alone and in combination demonstrated that the aVOR at least qualitatively exhibits vector summation. Recently, Gong et al. (2008) showed that responses to prosthetic stimuli delivered to electrodes implanted in 
coplanar canals on opposite sides of the head show evidence of linear summation. However, significant frequency- and amplitude-dependent nonlinearities are evident in aVOR responses of individuals with a single intact labyrinth (who most closely represent the functional goal of a vestibular prosthesis implanted unilaterally in a bilaterally vestibular-deficient individual; Carey et al. 2002a; Weber et al. 2008, 2009). The $3 \mathrm{D}$ aVOR may therefore be more complex than a static vector summation of different ampullary nerve inputs. Furthermore, nonlinearities could arise due to the dynamics of action potential initiation in ampullary nerve fibers spuriously excited or inhibited by current spread. Therefore, linear behavior of the electrically evoked 3D aVOR cannot be assumed.

In the present study, we assessed the linearity of the electrically evoked aVOR and then examined whether incorporating a linear precompensation encoding scheme in a multichannel vestibular prosthesis enables implanted animals to accurately sense and respond to sinusoidal head rotation about any specified 3D axis at any specified velocity within the range typical of normal behavior.

\section{METHODS}

\section{Subjects}

Adult wild-type 450-650 g chinchillas were used for all experiments, which were performed in accordance with a protocol approved by Johns Hopkins University Animal Care and Use Committee.

\section{Surgical procedures}

Surgical procedures were similar to those described previously in detail (Della Santina et al. 2007). Under general anesthesia (isoflurane, 3-5\%), a phenolic post was positioned on the skull in the midline, perpendicular to the mean horizontal semicircular canal plane and embedded in dental cement (ESPE ProTemp II, 3M Corp., Minneapolis, MN), forming a rigid connection to the skull. The post served to restrain the animal during aVOR testing. Electrodes were fashioned from pairs of Teflon-insulated $75-\mu \mathrm{m}$ Pt-Ir wire (Cooner Wire, Chatsworth, CA) for each of the canal electrodes and $125-\mu \mathrm{m}$ Pt-Ir wire for reference electrodes.

To provide reference data on the aVOR of an animal with a single normal labyrinth (against which to compare responses of bilaterally vestibular-deficient animals treated with unilateral prosthetic stimulation), three otherwise normal chinchillas were treated unilaterally via intratympanic injection of $\sim 0.5 \mathrm{~mL}$ gentamicin $(26.7 \mathrm{mg} / \mathrm{mL}$ buffered to $\mathrm{pH} 7.4$ with sodium bicarbonate) using a protocol described previously (Hirvonen et al. 2005). Head posts were placed subsequently as described above.

Electrodes were positioned in the left labyrinth for all four implanted chinchillas. The electrode for the anterior canal ampullary nerve stimulation was positioned via a fenestration in the internal auditory canal exposing the ampullary nerve just medial of the anterior canal ampulla. The electrode for horizontal canal stimulation was positioned via a $\sim 100-\mu \mathrm{m}$ fenestration on the anterolateral wall of the horizontal ampulla. The electrode intended for the posterior canal stimulation was inserted into a fenestration in the thin segment of the posterior canal and advanced toward the ampulla until resistance was encountered.

Two reference electrodes were implanted to provide a choice of two possible returns for the monopolar stimulation delivered via any of the stimulating electrodes. A distant return was inserted into neck musculature, and a near return was inserted into the common crus via a fenestration in the superior aspect of the anterior SCC. The distant return was stripped of approximately $5 \mathrm{~mm}$ of insulation, and the near return was stripped of approximately $1 \mathrm{~mm}$ of insulation. For each stimulating electrode, we selected the reference electrode that yielded the highest velocity aVOR response with lower axis misalignment.

At the time of electrode implantation, the semicircular canals of the right labyrinth were plugged with fascia and bone chips via fenestrations in their thin segments. In order to assess the impact of electrode placement alone, we did not plug the canals in the left labyrinth. Instead, the two animals (ch206 and ch207) that had residual aVOR after the surgery were treated with intratympanic gentamicin on the left side, which ablated all residual sensitivity to head rotation without prosthetic input in each case. (Intratympanic gentamicin kills type I vestibular hair cells and renders type II hair cells insensitive to endolymph motion (Della Santina et al. 2005c; Hirvonen et al. 2005; Lyford-Pike et al. 2007)).

\section{Eye movement measurement and analysis}

Real-time, binocular 3D video-oculography (3D VOG) was used for recording eye movements in response to head movement or electrical stimulation. The method we employed is analogous to that described in detail previously (Migliaccio et al. 2005; Della Santina et al. 2007), except for the use of faster cameras yielding a higher sample rate. The system was installed on a dual core 2-GHz 2-GB RAM PC running Windows XP. Firewire cameras (Dragonfly Express, Point Grey Research, Richmond, BC, Canada) retrofitted with 25.0-mm focal length, f/2.0 microvideo lenses were used to acquire $500 \times 400$ pixel, 8 -bit grayscale images at $180 \mathrm{~Hz}$ for each eye. An array of three fluorescent 
yellow $0.5 \times 0.5$-mm squares, separated by $0.5 \mathrm{~mm}$ and arranged in a $45^{\circ}$ right triangle on a black film, was placed on the topically anesthetized cornea of each eye using a small amount of veterinary tissue glue (e.g., VetBond, 3M Corp) after application of proparacaine and saline eye drops. The glue forms a conformal coat over the saline film on the cornea up to the limbus, making a translucent film that remains on the eye and passes under the lids during eye movement. A set of ultraviolet light-emitting diodes (UV LEDs) illuminated each marker, and a UV cut filter on each camera maximized contrast of the markers versus reflections of the LEDs on the conjunctiva. LabVIEW software (National Instruments, Austin, TX) was used to control camera settings, threshold the image, correct lens distortion, and determine the center of each marker using a center of mass algorithm. Each eye's center of rotation was aligned by centering a pattern of corneal reflections from LEDs attached to the camera at points equidistant from the lens axis.

Eye movement data were analyzed using custom software incorporating 3D rotational kinematics. Recordings from each eye indicating horizontal, vertical, and torsional eye rotations in eye coordinates were converted to 3D rotational position in rotation vector form in a right-hand-rule canal-referenced coordinate system. Velocity vectors were calculated from the corresponding rotation vectors, then filtered using a running spline interpolation filter (LabVIEW "Cubic Spline Fit' module with balance parameter 0.99995) applied to data linearly interpolated on a $1-\mathrm{kHz}$ time base. For eye responses to sinusoidal stimuli, each of the three eye movement components [horizontal, LARP (about the mean axis of the leftanterior/right-posterior SCCs), and RALP (rightanterior/left-posterior)] were separately averaged cycle by cycle for cycles free of saccades and blinks. LARP and RALP axes were approximated as being $45^{\circ}$ off the midline and in the mean plane of the horizontal SCCs.

\section{Motion stimulation}

Whole body rotations about mean SCC plane axes were used for testing aVOR responses to natural stimuli. Each animal was rigidly mounted in a gimbal bolted to a servo-controlled motor positioned so that the center of the animal's skull was aligned with the rotator's Earth-vertical axis. The gimbal was reoriented to bring each axis of interest coincident with the rotator's axis for each of three axes: horizontal, LARP (left ear down nose up) and RALP (right ear down nose up). The horizontal aVOR was tested with the gimbal in its reference position in which the animal's horizontal semicircular canal axes were approximately Earth-vertical. LabVIEW-based software controlled the motor. Sinusoids included rotations at $2 \mathrm{~Hz}$ at peak velocity $50^{\circ} / \mathrm{s}$. Stimulus duration was sufficient to include at least six cycles at each frequency, and only cycles not corrupted by blinks or other artifacts were analyzed.

\section{Electrical stimulation}

The complete dataset for each individual animal was obtained in a single session lasting approximately $3 \mathrm{~h}$. In all experiments, angular velocity of real or emulated head rotation about a semicircular canal's axis was used to modulate the pulse frequency of chargebalanced, symmetric, biphasic current pulses delivered to that canal's ampullary nerve. Pulse rate was modulated between 0 and $400 \mathrm{pps}$ at $2 \mathrm{~Hz}$ along a two-segment, piecewise-linear operating characteristic running linearly from 0 pps at $-300^{\circ} / \mathrm{s}$ (inhibitory head rotation for the left labyrinth) to $60 \mathrm{pps}$ (head stationary) then linearly from 60 to $400 \mathrm{pps}$ at $+300^{\circ} / \mathrm{s}$ (excitatory head rotation for the left labyrinth). We chose the 60-pps baseline stimulation rate based on preliminary experiments (Davidovics et al. 2009) that revealed much larger responses for lower baseline rates than for the supranormal baseline rates we (Della Santina et al. 2007) and others (Lewis et al. 2001, 2002; Merfeld et al. 2006) have used previously. This rate approximates the mean resting spike rate of vestibular afferent fibers in normal chinchillas (Baird et al. 1988; Hullar et al. 2005) and is about 50\% higher than the mean rate in labyrinths treated with intratympanic gentamicin (Della Santina et al. 2005c; Hirvonen et al. 2005).

The current amplitude of biphasic current pulses delivered at $350 \mu \mathrm{s} /$ phase and $1-\mathrm{Hz}$ modulation between 30 and 230 pps (half-maximal modulation) was slowly increased until signs of facial nerve stimulation were observed or an obvious shift in the eye response axis away from the target axis was detected, then lowered until these gross effects abated, typically resulting in a response amplitude of $25-50 \%$ s (amplitude of the best-fit single-frequency whole cycle sinusoid fit via single-frequency Fourier transform). The current amplitude and the return electrode used for monopolar stimulation delivered to the electrodes implanted in each canal are listed in Table 1. Charge per phase was kept constant for each animal once it was set during the preliminary stimulation session.

Stimulation current amplitude for each electrode was then downloaded to the multichannel vestibular prosthesis, which has been described in detail elsewhere (Della Santina et al. 2007). Pulse frequencymodulated biphasic pulse trains on the three electrodes were presented concurrently, but asynchronously, 


\section{TABLE 1}

Stimulation current levels ( $\mu \mathrm{A} /$ phase) and reference electrode used for each active electrode in each animal

\begin{tabular}{lccc}
\hline & \multicolumn{3}{l}{ Stimulation level $(\mu \mathrm{A} /$ phase) and reference electrode } \\
\cline { 2 - 4 } Animal ID & LA electrode & LH electrode & LP electrode \\
\hline ch205 & $120(\mathrm{CC})$ & $120(\mathrm{M})$ & $70(\mathrm{M})$ \\
ch206 & $350(\mathrm{CC})$ & $225(\mathrm{M})$ & $70(\mathrm{M})$ \\
ch207 & $140(\mathrm{CC})$ & $130(\mathrm{CC})$ & $170(\mathrm{CC})$ \\
ch208 & $35(\mathrm{CC})$ & $55(\mathrm{M})$ & $120(\mathrm{CC})$ \\
\hline
\end{tabular}

Amplitude and reference electrodes were determined for stimulating electrode and for each animal at the beginning of the recording session and kept constant for the duration of that experiment

$L A, L H$, and $L P$ active electrode's target ampullary nerve (left anterior, horizontal, and posterior, respectively), $M$ muscle reference electrode, $C C$ common crus reference

ensuring no overlap in pulse presentations. In the case of a timing conflict between the channels, pulses were queued up and presented in first-in/first-out order so that only one electrode was active at any moment.

To assay eye movement responses during a large variety of prosthetically encoded rotation angles in a precise and repeatable manner and to ensure that responses measured were solely due to prosthetic input, all subsequent experiments were performed with the head stationary. In place of the gyroscope inputs that are normally sampled by the prosthesis central processor, we substituted three analog signals generated by a USB-3103DAQ data acquisition card (Measurement Computing, Norton, MA) controlled by a PC running custom software written in LabVIEW.

\section{Assessment of linearity of electrically evoked aVOR}

To assess the degree of linearity for responses to stimuli targeting a given ampullary nerve, we measured eye rotation velocities during $2-\mathrm{Hz}$ sinusoidal modulation of the pulse rate at various stimulus intensities (SI) about baseline on that ampullary nerve's electrode, while pulse rates on electrodes targeting the other two ampullary nerves were kept constant at $60 \mathrm{pps}$. Changes in pulse rate encode head angular velocity, so SI is analogous to the amplitude of a sinusoidal head velocity. Down-modulating pulse rate from 60 toward 0 pps effectively encodes head rotation in the inhibitory direction, while up-modulating from 60 toward 400 pps encodes excitatory head rotation about the axis of the stimulated ampullary nerve's SCC. Using the piecewise linear mapping of the gyro-signal to pulse rate, SI of $0 \%$ delivered a steady 60 pps. SI of $50 \%$ modulated between 30 and 230 pps so that for half of the sinusoidal cycle, the pulse rate changed from 60 to 30 pps and back, and for the second half of the cycle, the pulse rate changed from 60 to 230 pps and back. SI of $100 \%$ modulated the pulse rate from 60 to 0 pps and back during the inhibitory half of the cycle and from 60 to 400 pps during the excitatory half of the cycle. Eye responses were recorded during sinusoidal modulation stimuli presented with SI of $20 \%, 40 \%, 60 \%, 80 \%$, and $100 \%$, in random order on each of the three electrodes.

\section{Finding parameters for precompensation matrix}

To determine the linear precompensation matrix for a given animal, we first tuned pulse current as described above, typically resulting in response velocities of approximately $25-50 \%$ s (amplitude of best-fit whole cycle sinusoid) for $50 \%$ SI on each of the three electrode channels activated individually. We then presented a series of stimulus combinations on each of the three active electrodes, with each triplet representing an axis of head rotation in a 3D space defined by the mean horizontal, LARP, and RALP semicircular canal axes. The stimulation vector comprised three parameters, each indicating the SI on each of the individual electrodes after precompensatory remapping. Sixty-five evenly spaced stimulus vectors spanning the space of possible rotation axes in $3 \mathrm{D}$ were initially presented at corresponding ratios of $50 \%$ SI about the 60-pps baseline on each electrode, typically producing aVOR response velocity amplitudes in the range $25-50^{\circ} / \mathrm{s}$. Using the complete set of aVOR velocity vectors in response to all commanded stimulus vectors, we then computed an optimal mapping matrix $\boldsymbol{M}$ for each animal using $\mathrm{QR}$ decomposition (Matlab, Mathworks, Cambridge, MA). This matrix described the least squares optimal linear 3D remapping relationship between the set of stimulus vectors and corresponding response vectors. The inverse of this matrix was then used for subsequent experiments to compute SI commands for each electrode given an arbitrary desired aVOR rotation axis and velocity. A similar approach was independently described in a recently awarded patent that included claims to the use of linear algebraic coordinate transformations in the setting of prosthetic vestibular stimulation (Merfeld 2008).

Preliminary experiments revealed that using SI other than $50 \%$ produced matrices which were similar to the one derived from using the SI of $50 \%$. For consistency, we used $50 \%$ SI to derive the optimal mapping matrix $\boldsymbol{M}$ for each of the animals used in these experiments.

\section{Assessing aVOR responses to precompensated stimuli}

To assay the response to precompensated prosthetic stimuli for a given desired head rotation axis, a ten- 
cycle burst of 2-Hz sinusoidal pulse frequency modulation was presented with the relative SI on each of the three active electrode channels set according to the appropriate output of Eq. 2. This was repeated in random order for 65 axes chosen to span a hemisphere at $22.5^{\circ}$ intervals. For each axis tested, stimulation was presented at different SI, corresponding to $12.5,25,37.5,50,75$, and $100^{\circ} / \mathrm{s}$ desired peak eye rotation response at $2 \mathrm{~Hz}$. Assuming a normal chinchilla aVOR gain of $\sim 0.4$, these correspond approximately to head rotations at peak velocity 32 , $62,94,125,188$, and $250^{\circ} / \mathrm{s}$. An analogous set of responses was measured on each animal using frequencies of $0.2,0.5,2$, and $5 \mathrm{~Hz}$ at $25^{\circ} / \mathrm{s}$ desired peak eye rotation velocity. The aVOR responses about each of the three spatial components (horizontal, LARP, and RALP) were measured in terms of the amplitudes of the best-fit whole cycle sinusoid at the same frequency as the stimulus. Measured aVOR responses were compared to the expected eye rotations, defined as being about the same axis as the intended virtual head rotation and with speed equal to that predicted for a normal chinchilla undergoing a true head rotation about the specified axis. We assumed a mean aVOR gain and phase of $0.5^{\circ}$ and $0^{\circ}$, respectively, for normal chinchillas at $2 \mathrm{~Hz}$ (Della Santina et al. 2007).

\section{Statistical methods}

Linearity of single-channel responses was quantified using the Pearson correlation coefficient for a least mean square error linear fit. To determine whether misalignment and error in eye velocity were significantly different between the uncompensated and precompensated paradigms, we used a multivariate general linear model constructed and analyzed using SPSS 16.0 software (SPSS Inc., Chicago IL).

\section{RESULTS}

When a normal chinchilla is rotated sinusoidally at $2 \mathrm{~Hz}$ and $50^{\circ} / \mathrm{s}$ peak about the mean axis of each coplanar pair of semicircular canals, normal aVOR responses are only partially compensatory for the head rotation, with the resulting eye movements being opposite the direction of head rotation but at only about half the head velocity (Fig. 1A, column 1 ). After unilateral gentamicin treatment, responses to sinusoidal head rotation become asymmetric, with a near-normal aVOR response during rotation toward the normal ear and attenuated response during ipsilesional rotation (Fig. 1A, column 2). After canal plugging, electrode insertion, and gentamicin treatment resulting in bilateral vestibular deficiency, no aVOR response is observed for head rotation in either direction (Fig. 1A, column 3). When the same animal (ch207) is electrically stimulated at $50 \%$ SI on individual SCCs' electrodes, current spread to nontargeted branches of the vestibular nerve is evident in that the aVOR response axis does not align well with the intended axis (especially for that RALP case of Fig. 1A, column 4). Precompensatory adjustment of the stimuli attenuated the undesired response components and normalized both the axis and amplitude of the response (Fig. 1A, column 5). Asymmetry was observed in the responses for all four animals, indicating that unilateral prosthetic stimulation restores a bilaterally vestibular-deficient animal to a state similar that of an animal with a single normal labyrinth (i.e., Fig. 1A, column 2).

Similar improvement in the axis alignment was evident for all four experimental animals when we compared observed and actual axes of aVOR response for modulation of each SCC's electrode individually (Fig. 1B).

To assess whether the relationship between singlechannel SCC stimulation intensity and aVOR response velocity is sufficiently linear to justify use of a linear precompensation scheme, we assayed aVOR response amplitude for each animal as a function of SI amplitude (Fig. 2). The Pearson correlation coefficient for a linear fit between the SI amplitudes and the corresponding aVOR response amplitudes was $>0.93$ in all cases.

Observed aVOR responses matched desired amplitude and axis better when precompensation was used to correct for axis misalignment than did responses to stimuli presented without precompensation. Figure 3 compares desired and actual aVOR responses for 16 stimulus vectors without (panel A) and with precompensation (right panel B). In the first case, stimuli without precompensation were presented at $50 \%$ SI. In the second case, precompensation was used to correct for axis misalignment and to specify desired $25^{\circ} / \mathrm{s}$ peak eye velocities. Misalignment is clearly reduced by precompensation. This reduction of misalignment is also apparent in (Fig. 3C, D), which shows misalignment without and with precompensation for all 65 head rotation axes emulated during the experiment. Precompensation also reduced errors in the speed of eye movement responses (Fig. 3E, F).

To discern whether animal tested, order of stimulus presentation, axis of desired response, and/or combinations of these factors significantly affected misalignment and amplitude error of responses, these factors were included in the multivariate general linear model as independent variables. Order of presentation was defined by binning data acquired within each of two test sessions (either uncompensated at $50 \%$ SI and $2 \mathrm{~Hz}$ or precompensated at $25^{\circ} / \mathrm{s}$ desired velocity and $2 \mathrm{~Hz}$ ), each of which comprised 


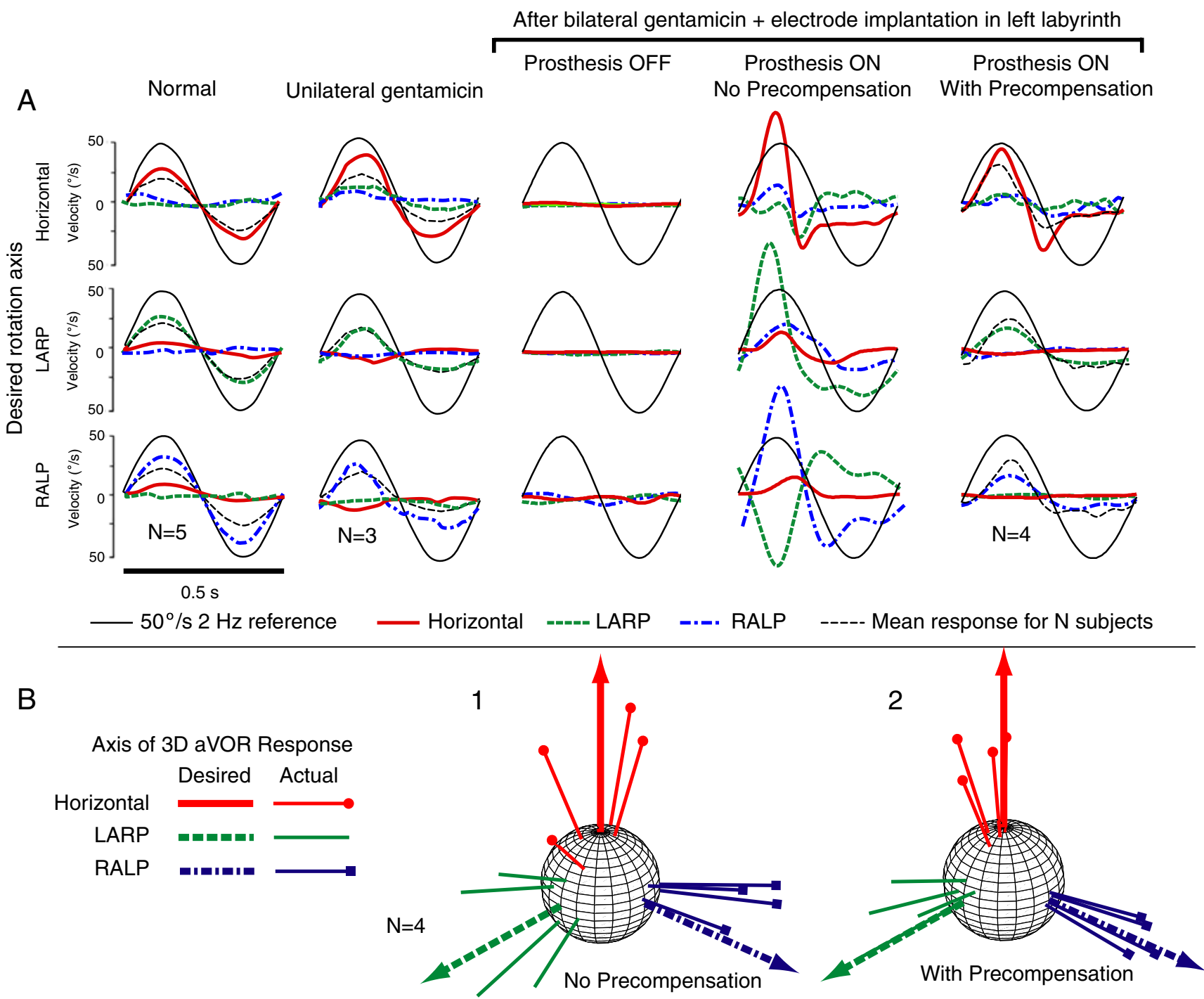

FIG. 1. A Precompensatory 3D remapping improves both axis and amplitude of prosthesis-encoded head velocity percept. Column 1 Mean head and eye velocity for a normal chinchilla during $50 \% \mathrm{~s}$ peak $2-\mathrm{Hz}$ whole body rotations in darkness about the axis of each coplanar pair of semicircular canals (SCC): horizontal (top row), left anterior/right posterior ( $L A R P$, middle row), and right anterior/left posterior (RALP, bottom row). For clarity, only mean responses are shown; the standard deviation for each trace was $<6 \%$ s. Eye traces are inverted to compare with head. Normal chinchillas exhibit good alignment with head rotation axis and symmetric gain (eye velocity/ head velocity) of about 0.5 (data from Della Santina et al. 2007). Dashed curves indicate mean responses for all animals studied. Column 2 aVOR responses for chinchillas treated with intratympanic

measurements of 65 different desired axes presented in random order divided into ten epochs. The first epoch comprised responses from the first ten presentations, the second epoch had the responses from the second ten, and so on. Desired eye rotation axis was similarly binned into four sectors of space to determine whether misalignment and velocity error varied systematically with desired axis.

Responses to precompensated stimuli were significantly better than uncompensated responses, inde- gentamicin (ITG) in one ear are attenuated during ipsilesional rotation. Column 3 Head rotation of a bilaterally vestibular-deficient animal (ch207) after ITG and canal plugging elicits no response for any axis when prosthesis is off. Column 4 Responses for the same animal when pulse rates on individual electrodes implanted in the left labyrinth SCCs are modulated without precompensatory $3 D$ remapping. Misalignment due to current spread is evident for all three axes of rotation. Column 5 aVOR responses of the same animal to stimuli with precompensatory $3 D$ remapping are like those of animals with a single working labyrinth (column 2). B 3D aVOR responses to prosthetic stimuli encoding head rotation about each of the three SCC axes without (B1) and with (B2) precompensatory 3D remapping for each of four implanted animals.

pendent of all other factors, yielding smaller misalignment $(p<0.001)$ and smaller error in eye velocity amplitude $(p<0.001)$. This was true for every animal, although differences between animals were significant for both misalignment and velocity error $(p<$ 0.001 ). Order of presentation had no significant effect alone on misalignment or velocity error $(p=0.91$ and 0.55 , respectively), nor did it have any significant effect in combination with other factors, suggesting that adaptation and central compensation did not occur to 

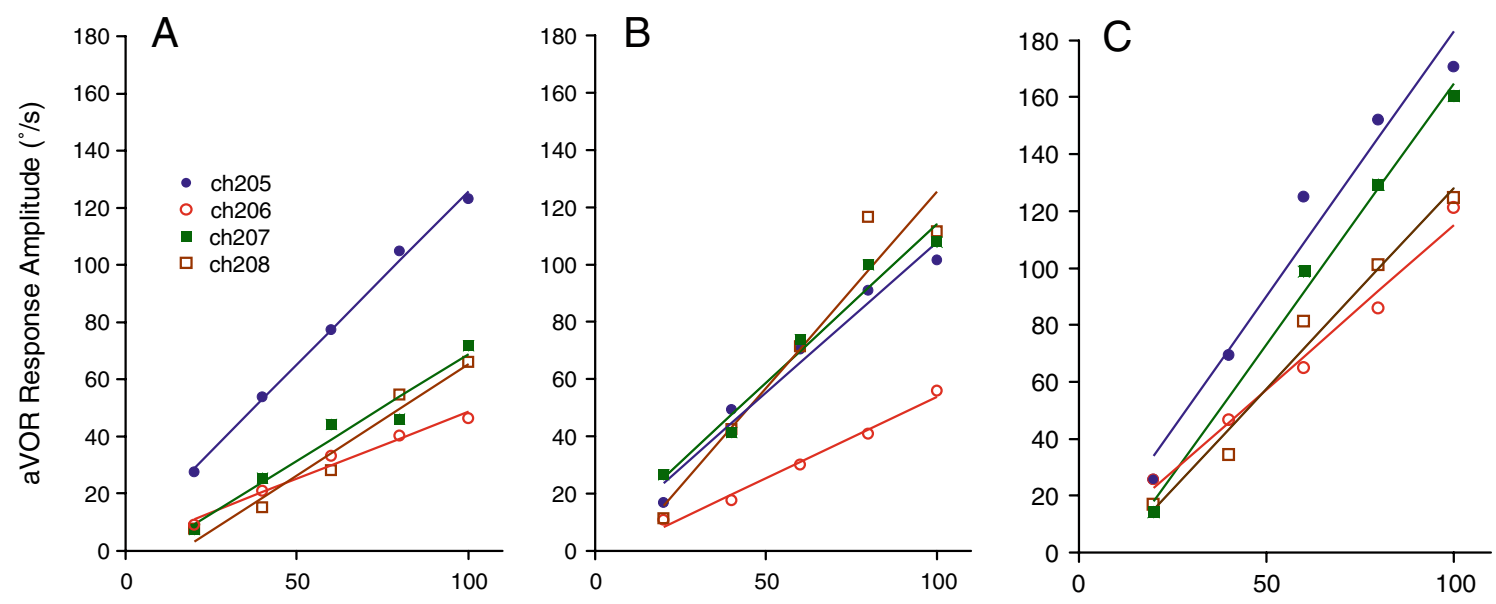

Stimulus Intensity (\% of maximal pulse rate modulation)

FIG. 2. Amplitude of best-fit whole cycle sinusoid velocity of aVOR response to stimuli delivered on an electrode near the horizontal (A), anterior (B), and posterior $(\mathbf{C})$ semicircular canals of the left labyrinth in each of four chinchillas. Velocity varies almost linearly with stimulus intensity (pulse frequency depth of modulation as defined in text). $R^{2}>0.93$ for linear fit in each case.

a substantial degree during an experiment. The 3D orientation of the desired eye rotation axis (i.e., the inverse of the head rotation axis being encoded) did not significantly affect misalignment $(p=0.21)$, but did have a significant effect on velocity error $(p<0.001)$, which was lowest for desired eye rotation axes within the sector for which inputs to all three SCCs were excitatory. This reflects asymmetry of responses to unilateral prosthetic stimulation, which are evident in Fig. 1A, column 5.

Pooling responses to all 65 vectors over a range of simulated head velocities for each of the four animals, the misalignment error between the desired axes of rotations and the corresponding aVOR responses was $27.5 \pm 14.4^{\circ}($ mean $\pm \mathrm{SD})$ for the $50 \%$ SI stimuli prior to precompensation. After precompensation, misalignment decreased to $16.5 \pm 9.6^{\circ}$. For each animal, misalignment and velocity errors were lower with precompensation than without. This reduction in error was most pronounced for stimuli representing faster head rotations (Fig. 4A).

For each of the animals, the aVOR response velocity after precompensation increased as expected with increases in the desired aVOR velocity (Fig. 4B). Pearson correlation coefficient for a linear fit between the desired and recorded aVOR response amplitudes was 0.8014 .

Response dynamics evaluated for all 65 vectors spanning the possible 3D head rotation axes for each of four frequencies $(0.2,0.5,2$, and $5 \mathrm{~Hz})$ at $25^{\circ} / \mathrm{s}$ desired eye velocity amplitude showed a high-pass characteristic somewhat similar to that of the normal aVOR (Della Santina et al. 2007; Fig. 4C). The cutoff frequency, $f_{c}$, for a least squares fit of a single-order high-pass filter to the frequency response data in Fig. 4C (averaged across all animals in decibels at each frequency) was $0.42 \mathrm{~Hz}$, corresponding to a time constant, $\tau$, of $0.38 \mathrm{~s}$. This is between the values we observed for normal chinchillas $\left(f_{\mathrm{c}} \sim 0.05-0.1 \mathrm{~Hz} ; \tau \sim\right.$ 1.6-3.2 s) and prosthetically stimulated animals $\left(f_{\mathrm{c}} \sim\right.$ $2 \mathrm{~Hz} ; \tau \sim 0.08 \mathrm{~s}$ ) in our previous study (Della Santina et al. 2007). It is different from values reported previously for normal chinchillas by Merwin et al. (1989) $\left(f_{\mathrm{c}} \sim 0.02 \mathrm{~Hz} ; \tau \sim 7.5 \mathrm{~s}\right)$. However, the present study's result must be considered in light of variability across animals, asymmetry of responses, and the fact that because our focus here was on eye movement alignment (which was evaluated in each case for multiple replicates of 65 different directions), the evaluation was repeated for only four frequencies in the range for which aVOR dominates gaze stabilization. None of those frequencies tested was low enough to unambiguously identify an inflection point in the frequency responses. Comparison to frequency response data from Gong et al. for the electrically evoked aVOR in guinea pig is complicated by their use of response normalization and a high-pass filter to condition gyro output signals (Gong and Merfeld 2002); however, their data revealed a similar high-pass shape, $f_{\mathrm{c}} \sim 0.5 \mathrm{~Hz}$ and a somewhat steeper fall in gain with decreasing frequency.

\section{DISCUSSION}

Our observations confirm that prosthetically evoked aVOR responses exhibit linearity and vector summation to sufficient extent that a precompensation strategy incorporating a linear coordinate system transformation computed from a set of desired and actual response amplitudes can significantly correct misalignment of 3D aVOR responses (and presumably 


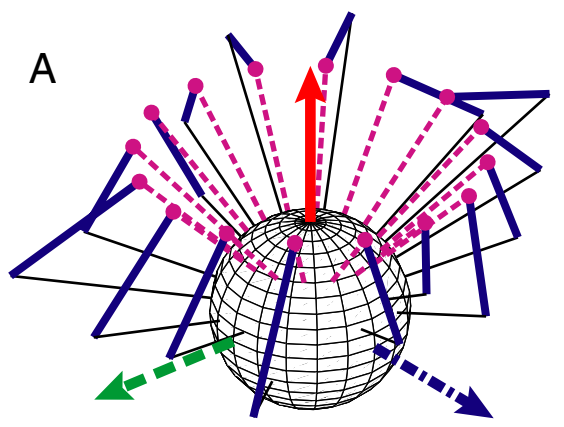

\section{---๑ Desired aVOR velocity Actual \\ Error (Actual-Desired)}

Reference Axes

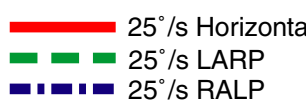

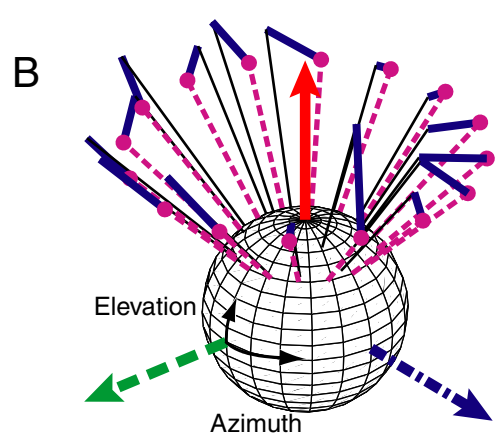
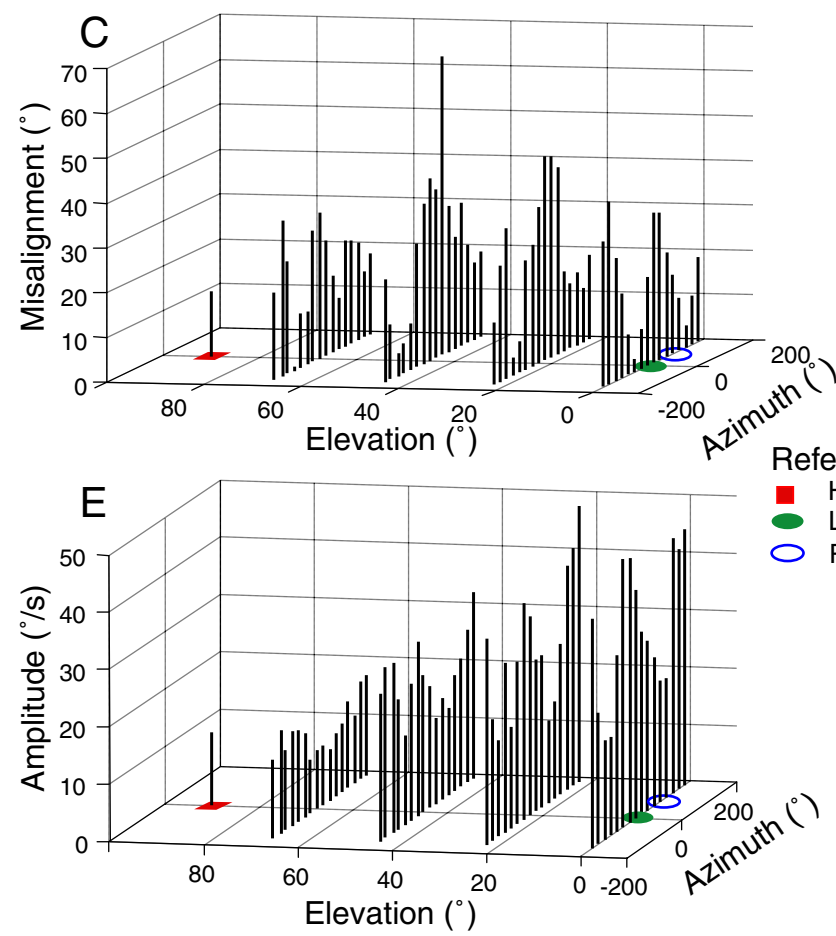

FIG. 3. When precompensatory $3 \mathrm{D}$ remapping is employed, axis and speed of observed aVOR eye rotation responses align better with desired responses for virtual head rotations about any $3 \mathrm{D}$ axis as compared to the responses to uncompensated rotations. A Desired and actual eye velocity for 16 different directions tested prior to precompensation, each at $2 \mathrm{~Hz}$ and $50 \%$ stimulus intensity ( $S I$, defined in text). Axis and speed are represented by orientation and length, respectively, of plotted vectors. Dashed lines define a headbased coordinate system comprising the horizontal, left anterior/right posterior $(L A R P)$ and right anterior/left posterior $(R A L P)$ axes; the length of each denotes $50 \%$ s peak head velocity which would evoke

of head movement percepts) that would otherwise occur due to current spread from each electrode to axons outside its target nerve branch. This optimal transformation, which effectively encodes both a reorientation and a rescaling of stimulus intensities, significantly corrected both the axis and amplitude of eye rotational velocity, with the degree of correction greatest at the largest amplitudes tested. Incorporating this correction allowed us to maintain 3D aVOR alignment while reliably achieving larger peak eye
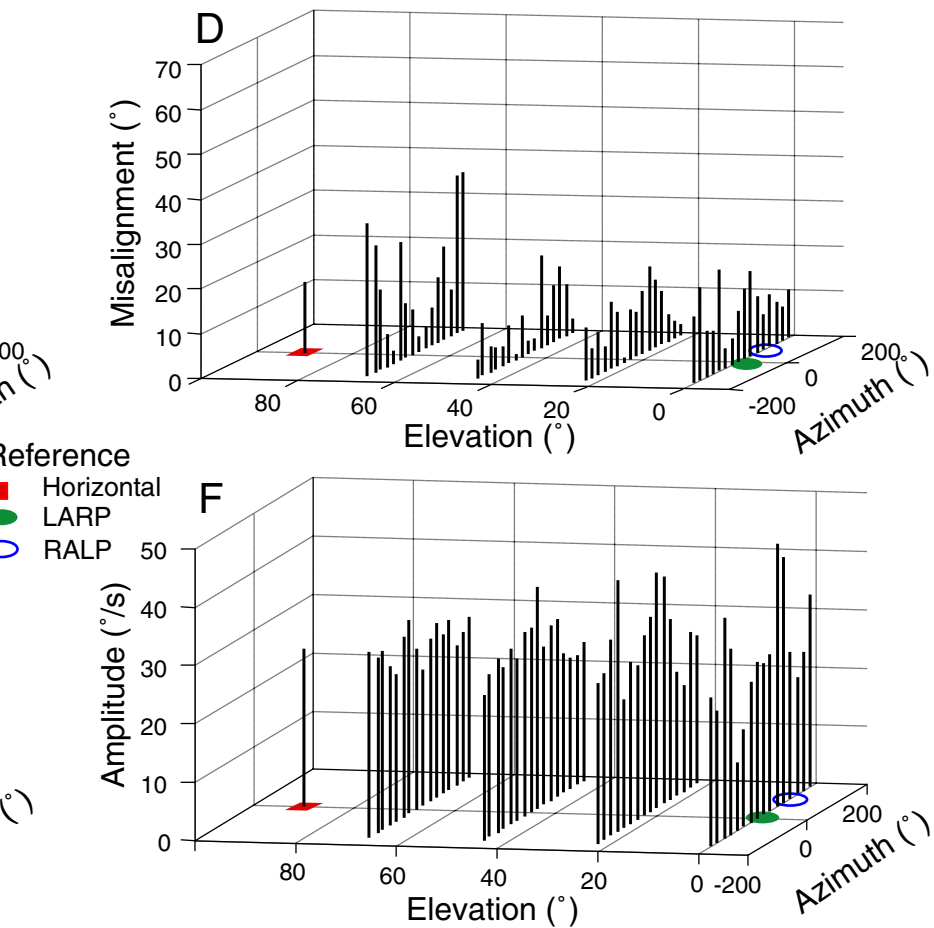

an eye velocity of approximately $25 \% \mathrm{~s}$ in a normal chinchilla. B Results for the same 16 desired rotations after precompensation (again $2 \mathrm{~Hz}$, desired peak velocity $25 \%$ s). Thick lines indicate error between response and stimulus. Errors are significantly smaller after precompensation. C Misalignment (angle between desired and actual eye rotation axis) for each of the 65 virtual head rotations tested without precompensation. Azimuth and elevation of each axis is as defined in B. D Misalignment is significantly smaller after precompensation. E, F Peak velocity for same cases as in $\mathbf{C}$ and $\mathbf{D}$. Responses to precompensated stimuli are significantly closer to the desired velocity of $25^{\circ} / \mathrm{s}$

movement response velocities than we and others have reported previously (Gong and Merfeld 2000; Lewis et al. 2001, 2002; Della Santina et al. 2005a, b; Merfeld et al. 2006, 2007; Della Santina et al. 2007; Gong et al. 2008; Lewis et al. 2008, 2009). Responses to unilateral prosthetic stimulation exhibited excitation-inhibition asymmetry both before and after precompensation, similar to responses of animals with a single normal labyrinth experiencing natural head rotation stimuli. 

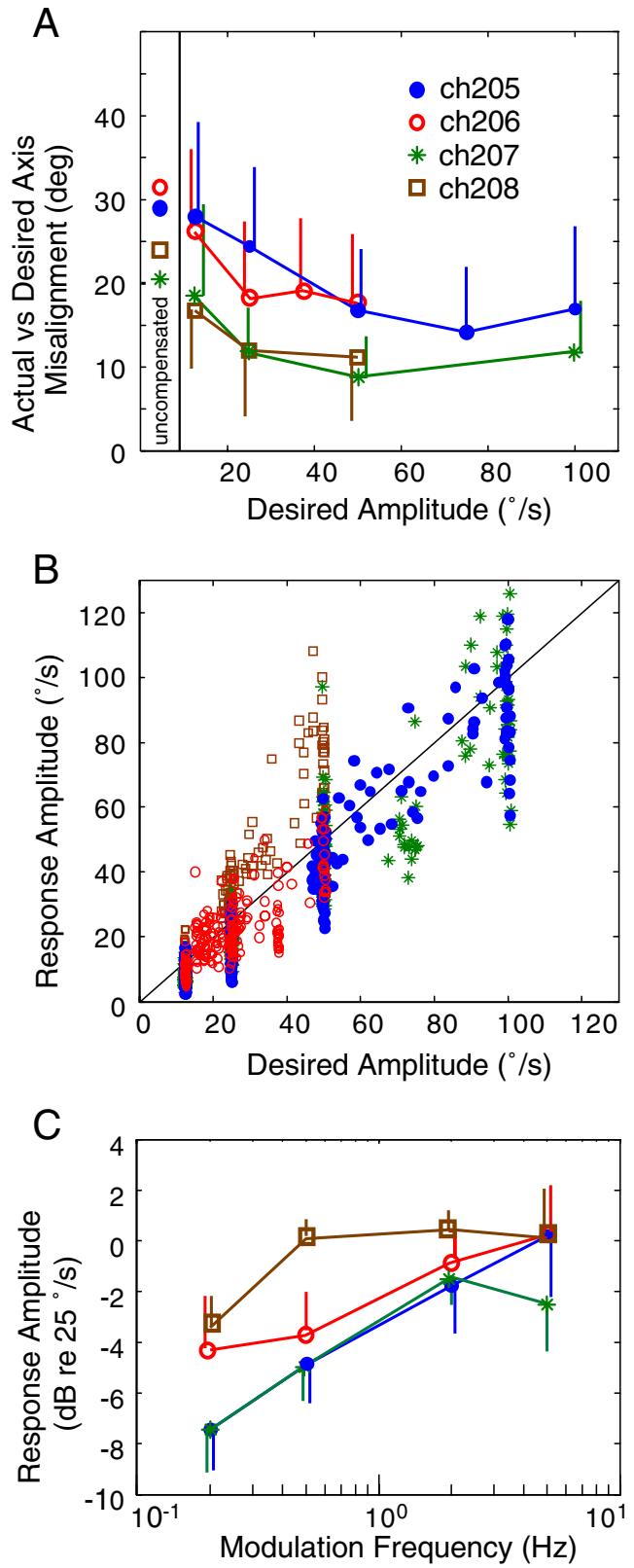

FIG. 4. A Misalignment angle for aVOR responses to prosthetic input is less with precompensation than without and smallest for high velocity head rotation. B The relationship between desired and observed aVOR responses with precompensation clusters around equality for all four implanted animals. C As for normal animals (Della Santina et al. 2007), a high-pass characteristic is noted for each animal's aVOR response as a function of the modulation frequency encoding virtual $50 \% \mathrm{~s}$ head rotations.

Asymmetry of responses to unilateral prosthetic stimulation

Prior studies of prosthetic stimulation using unilaterally implanted, head-mounted devices have tried to achieve symmetric eye movement responses to sinusoidal head rotations by modulating stimuli about a baseline rate that is well above that of most normal vestibular afferents. For example, we previously used baseline rates of $100 \mathrm{pps}$ in chinchillas (Della Santina et al. 2005a, b, 2007), while Gong et al. have used baseline rates of 150 pps in guinea pigs (Gong and Merfeld 2000), 200 pps in squirrel monkeys (Gong et al. 2008), and 250 pps in rhesus monkeys (Lewis et al. 2008), greater than or equal to two times the mean resting rate of normal vestibular afferents in each case. The rationale underlying this has been to expand the dynamic range over which a unilaterally implanted prosthesis can use downward modulation of pulse rate to encode inhibitory head rotations.

We departed from that approach in the present study, choosing instead a lower baseline pulse rate closer to spontaneous discharges rates measured in normal vestibular afferents. This change was motivated by our recent observation that while supernormal baseline rates result in roughly symmetric responses, those responses are almost invariably smaller in amplitude than those we can achieve when using baseline rates that better approximate the mean spontaneous firing rate of normal vestibular afferents. A baseline rate of 60 pps was selected based on results of a previous study of chinchillas treated unilaterally with intratympanic gentamicin that found spontaneous rates of $55 \pm 20$ (mean $\pm \mathrm{SD}$ ) spike/s for 62 control side vestibular afferent fibers and $36 \pm 15$ for 122 treated side afferents (Della Santina et al. 2005c), consistent with prior studies by others (Hullar and Minor 1999; Hirvonen et al. 2005).

Using this lower baseline rate has reliably resulted in peak excitatory responses exceeding $100^{\circ} / \mathrm{s}$ (Fig. 4B). While lower baseline rates leave less room for encoding inhibitory head rotation and thus result in an obvious excitation-inhibition asymmetry (e.g., Fig. 1A, column 5), the degree of asymmetry is similar to that observed in animals that have one normal labyrinth and one gentamicin-treated labyrinth (Fig. 1A, column 2; also see (Jones et al. 2003; Della Santina et al. 2005c)). Since the latter animals represent our goal with a unilateral prosthesis (i.e., to restore one implanted labyrinth to near-normal function after bilateral vestibular sensory loss), we consider this degree of asymmetry acceptable. Clinical experience demonstrates that patients with one remaining normal labyrinth typically compensate very well after unilateral gentamicin treatment (Carey et al. 2002b) or labyrinthectomy (e.g., Diaz et al. 2007), ultimately becoming essentially asymptomatic during most head movements typical of daily life. This occurs despite the fact that afferent firing rates do not rise to supernormal levels in the intact labyrinth after contralateral gentamicin treatment (Hullar and Minor 1999; Della Santina et al. 2005c; Hirvonen et al. 2005) or labyrinthectomy (Sadeghi et al. 2007a).

We used single-valued measures of best-fit whole cycle sinusoid amplitude for stimulus intensity and 
response velocity rather than half-cycle measures because we sought to design a single optimal precompensation matrix for each animal. Excitationinhibition asymmetry was explicitly incorporated via the piecewise-linear, two-segment mapping from gyro input to pulse rate on the corresponding electrode channel. As evident from Fig. 3, this approach was successful in reducing misalignment and correcting amplitude. It might be possible to improve response alignment and amplitude fidelity even further by devising a more elaborate scheme that involves different matrices for excitatory and inhibitory inputs. However, using different matrices for excitatory and inhibitory inputs would be cumbersome, because one could then require eight matrices to account for all possible combinations of excitatory and inhibitory inputs on three gyro input channels.

The ideal shape of the mapping from gyro input to pulse rate has yet to be determined, and further optimization to maximize both response amplitude and symmetry might be possible. Apart from yielding large-amplitude responses with asymmetry similar to that observed during natural stimulation of animals with a single normal labyrinth, the simple piecewiselinear mapping used in the present study allowed us to examine the linearity of physiologic responses without the potential confounding effect of the multiple degrees of freedom available when specifying the shape of a sigmoidal mapping curve.

\section{Key differences between natural and prosthetic ampullary nerve stimulation}

Each normal ampullary nerve contains $\sim 1,500$ asynchronously firing afferent fibers (Boord and Rasmussen 1958) with a diversity of spontaneous rates, baseline firing rate variability, thresholds, sensitivities, excitation/inhibition asymmetries, and terminal locations in the cristae (Goldberg 2000). This allows an ampullary nerve to convey much more information than could be conveyed by a single axon. For example, a group of afferents with a wide range of thresholds and sensitivities can provide a quasi-linear ensemble signal over a wide dynamic range of head velocities. Similarly, afferents with different response dynamics (e.g., due to crista location and morphology of their synapses with hair cells) can encode different frequency components of transient and sustained head movements. Stochastic independence between fibers further increases the amount of information carried by the ensemble (Sadeghi et al. 2007b).

In contrast, pulsatile prosthetic stimuli like those we currently use probably evoke nearly synchronous action potentials in all afferent fibers for which the pulse is above threshold. Our present inability to controllably excite different subpopulations of axons that normally have different thresholds for head rotation probably restricts the dynamic range over which we can accurately encode head velocity. The pattern of fiber activity presented to vestibular nuclei is probably further distorted by the fact that fibers with irregular spontaneous discharge are more sensitive to applied currents than are regular fibers (Goldberg et al. 1984), and larger caliber axons tend to be more sensitive to applied current than are thinner fibers. Irregular, large-caliber fibers tend to terminate in calyceal synapses with type I hair cells in the central zone of the crista, and their natural responses tend to be more phasic (i.e., more nearly in phase with head acceleration than velocity) and less linear than those of regular afferents terminating on boutons. Thus, nearthreshold prosthetic electrical stimuli might initially be "interpreted" by vestibular nuclei as representing a head rotation with different frequency content than that measured by the gyros. Refinements of pulse shape may help us semi-selectively exploit differences in activation dynamics of different classes of fibers, but for now, the signal we can present to the brainstem is a relatively crude facsimile of normal.

Another difference between the prosthetic stimuli we provide and sensory signals reported by a single normal labyrinth during natural head movement is that prosthetic/electrical input to targeted ampullary nerves may be incongruent with activity (or lack thereof) on the ipsilateral macular nerves. For example, during a head roll about an Earth-horizontal axis (EHA), aVOR responses to ampullary nerve activity are normally augmented by responses to macular nerve inputs driven by the utricle and saccule. This effect is most prominent for low-frequency EHA movements (as shown by Dickman et al. 2000 for pigeon and Hess and Angelaki 1997 for rhesus monkeys), while canal-mediated components dominate for all Earth-vertical axis (EVA) rotations and for EHA rotations at frequencies above about $0.1 \mathrm{~Hz}$. Otolith-mediated contributions were probably minimal for the data we present, because all our stimuli were $\geq 0.2 \mathrm{~Hz}$, all our mechanical stimuli were EVA, and we kept animals stationary during electrical-only stimuli. However, an animal wandering around in its cage while using a prosthesis would experience some EHA rotations at $<0.1 \mathrm{~Hz}$. Under those conditions, lack of macular nerve modulation during EHA lowfrequency rotations would be incongruent with prosthetic ampullary nerve stimuli (and conversely, any residual otolith endorgan mechanosensitivity would probably augment the aVOR).

Our data confirm that congruence among all ampullary and macular nerves is not a prerequisite for eliciting an aVOR sufficient to stabilize gaze, at least over the frequency range for which the aVOR is most essential for maintaining stable gaze. Many 
examples of nystagmus elicited by incongruent labyrinthine inputs exist (e.g., benign paroxysmal positional vertigo; Tullio phenomenon in superior canal dehiscence; unilateral stimulation studies by Ewald, Cohen, Suzuki, and others; see Carey and Della Santina 2005 for review). In the case of the prosthetic electrical stimulation experiments presented here, the unimplanted labyrinth is not reporting any movement at all (via canals or otolith endorgans) in a stationary animal receiving prosthetic electrical stimuli via one implanted ear, yet this did not preclude eliciting VOR responses that align well with the 65 desired axes we tested. Therefore, we conclude that congruent mechanical input via the contralateral macular nerves is not needed to evoke VOR responses that can stabilize gaze.

How ipsilateral macular nerve input is integrated with prosthetically evoked ampullary nerve input is an interesting question. Using the prosthesis should allow partial decoupling of parts of the labyrinth to facilitate answering this question in future studies. To the extent that electrical stimulation intended for ampullary nerves spreads to excite the utricular and/ or saccular nerves, the compensation procedure we used could be extended to leverage that current spread and improve misalignment during Earthhorizontal rotations.

Fortunately, neural signal pathways mediating the vestibulo-ocular reflex are highly adaptive, and prosthetic electrical stimulation can clearly elicit aVOR responses similar to those evoked by a normal labyrinth over a fairly wide range of head velocity and frequency. Nonetheless, we continue exploring approaches to optimization of stimulus encoding to reduce the burden on central compensatory mechanisms and expand the dynamic range over which prosthetic stimuli can accurately encode head rotation (Davidovics et al. 2010)

\section{Translating the approach toward human application}

Although linear precompensation works well to correct alignment and amplitude of the electrically evoked 3D aVOR in chinchillas, the extent to which this approach will be necessary and effective when vestibular prostheses are applied to humans is difficult to predict with certainty. Distances between neighboring ampullary nerves in the human labyrinth are larger than those in chinchillas, while precision of surgical positioning should be about the same, so the amount of current spread to non-target nerve branches might be less in humans and might be low enough that precompensation becomes unnecessary. The few data available are inconclusive: recent attempts to selectively stimulate the posterior ampul- lary nerve intraoperatively in alert humans apparently did not result in eye rotations purely about the desired axis (Wall et al. 2007), whereas the posterior ampullary nerve is usually the easiest branch of the chinchilla vestibular nerve to stimulate intensely with high selectivity and low misalignment. Tests of vestibular prosthetic stimulation in rhesus monkeys, which are intermediate in size between chinchillas and humans, suggest that misalignment may be slightly better than in chinchillas implanted using similar techniques (Lewis et al. 2009; Dai et al. 2010).

Years of clinical and research experience with cochlear implants suggest that the success of vestibular prostheses intended for human use will depend on multiple other factors. Cochlear implant users vary widely in their ability to perceive speech (Firszt et al. 2004; Zeng 2004; Wilson and Dorman 2008). Much of that variability is apparently due to differences in the survival and plasticity of neural substrates available to electrodes (e.g., cochlear nerve axons, the cochlear nucleus neurons upon which they synapse, and other brainstem and midbrain nuceli en route to auditory cortex), prompting efforts to preserve those substrates through anticipatory pharmacologic protection from ototoxic agents (Chen et al. 2007); intralabyrinthine delivery of growth factors, stem cells, and gene therapy (Hendricks et al. 2008); and, when no better alternative exists, prompt implantation and delivery of neuroprotective prosthetic input (Ryugo et al. 2005; Stakhovskaya et al. 2008).

Beyond optimizing the neuronal milieu, multiple design and rehabilitative techniques might be enlisted to further optimize responses to prosthetic stimulation beyond the results achieved using linear algebraic precompensation. These include:

1. Improvements in electrode design and surgical technique could help place cochlear electrodes nearer to their neural targets and further from neighboring axons (e.g., Gstoettner et al. 2001; Middlebrooks and Snyder 2007). Similar efforts may help optimize electrode-nerve coupling for vestibular prostheses.

2. Multipolar stimulation paradigms can provide more selective cochlear nerve stimulation by "steering" the current toward target axons between adjacent electrodes (Bonham and Litvak 2008); a similar approach may help to steer current toward one ampullary nerve and away from another (Chiang et al. 2009).

3. Optimization of stimulation parameters (e.g., pulse shapes, durations and intensities) might exploit the differential sensitivity of irregular and regular vestibular afferent fibers to electrical stimuli, allowing implant designers better control over the constellation of activity delivered to vestibular 
nuclei by different classes of vestibular afferent (Gorman and Mortimer 1983; Goldberg et al. 1984; Goldberg 2000; Merrill et al. 2005). If it can be achieved, semi-selectively addressing irregular afferents and regular fibers via different pulse shapes or amplitudes might enable the prosthesis to better encode quick, transient head rotations.

4. Cross-axis adaptation mechanisms should help trim up responses to prosthetic vestibular nerve stimuli distorted by spurious stimulation of non-target branches. Multiple studies suggest this should occur but also demonstrate that there are limits to cross-axis adaptation (e.g., Hay 1968; Miles and Fuller 1974; Miles et al. 1980; Viirre et al. 1998; Ma et al. 2000; Lewis et al. 2001). The closer we can bring the initial response to our desired outcome, the more likely it is that central adaptive mechanisms can finish the job (Schubert et al. 2008). Vestibular rehabilitation exercises enhance vestibular compensation after unilateral labyrinthine injury by enlisting adaptive vestibulocerebellar circuits to improve gaze and postural stability (Szturm et al. 1994; Scherer et al. 2008; Schubert et al. 2008). After unilateral labyrinthectomy, the central nervous system is quite adept at asymmetric adaptation during visuo-vestibular conflict exercises designed to augment aVOR gain during head rotations toward the deficient side (Ushio et al. 2009). Analogous exercise regimens might be harnessed to help implant users to adapt to prosthetic input.

5. In the work presented here, we focused on creating a single compensation matrix based on the best-fit whole cycle sinusoid. While the aVOR responses to precompensated stimulation clearly show reduced misalignment between the desired and actual responses, it may be possible to reduce this error further by taking into account the response asymmetry between the excitatory and inhibitory phases of sinusoidal stimulation. This could be accomplished in future studies by creating more elaborate precompensation scheme based on two sets of coefficients in which one set would correspond to the aVOR responses to excitatory stimulation delivered to a particular electrode and the second to the inhibitory stimulation. Further experiments that investigate adaptation to chronic stimulation should elucidate the need for developing a more complex precompensation scheme.

Developers of vestibular prostheses may need to enlist all of the approaches above to achieve the desired result of restoring gaze stability and more normal perception of head movement to individuals disabled by bilateral loss of labyrinthine function.

\section{ACKNOWLEDGMENTS}

This work was supported by the National Institute on Deafness and Other Communication Disorders (R01DC009255, K08DC006216 and F32DC009917, F31DC010099) and the American Otological Society. The authors thank Paul Fuchs PhD for comments on an earlier version of this manuscript. Dr. Migliaccio's current address is Balance and Vision Laboratory (Rm G26), Prince of Wales Medical Research Institute Cnr Barker Street \& Easy Street Randwick Australia 2031.

\section{REFERENCES}

Aw ST, Haslwanter T, Halmagy GM, Gurthoys IS, Yavor RA, Todd MJ (1996a) Three-dimensional vector analysis of the human vestibuloocular reflex in response to high-acceleration head rotations. I. Responses in normal subjects. J Neurophysiol 76:4009-4020

Aw ST, Halmagyi GM, Haslwanter T, Curthoys IS, Yavor RA, Todd MJ (1996b) Three-dimensional vector analysis of the human vestibuloocular reflex in response to high-acceleration head rotations. II. Responses in subjects with unilateral vestibular loss and selective semicircular canal occlusion. J Neurophysiol 76:4021-4030

Baird RA, Desmadryl G, Fernandez C, Goldberg JM (1988) The vestibular nerve of the chinchilla. 2. Relation between afferent response properties and peripheral innervation patterns in the semicircular canals. J Neurophysiol 60:182-203

Black FO, WADE SW, NASHNer LM (1996) What is the minimal vestibular function required for compensation? Am J Otol 17:401-409

Bonham BH, Litvak LM (2008) Current focusing and steering: modeling, physiology, and psychophysics. Hear Res 242:141-153

BOORD RL, RASMUSSEN GL (1958) Analysis of the myelinated fibers of the acoustic nerve of the chinchilla. Anat Rec 130:394

Carey JP, Della Santina CC (2005) Principles of applied vestibular physiology. In: Cummings CW (ed) Otolaryngology-head \& neck surgery. Elsevier, Amsterdam, pp 3115-3159

Carey JP, Minor lB, Peng GCY, Della Santina CC, Cremer PD, Haslwanter T (2002a) Changes in the three-dimensional angular vestibulo-ocular reflex following intratympanic gentamicin for Meniere's disease. JARO 3:430-443

Carey JP, Hirvonen T, Peng GC, Della Santina CC, Cremer PD, Haslwanter T, Minor LB (2002b) Changes in the angular vestibulo-ocular reflex after a single dose of intratympanic gentamicin for Meniere's disease. Ann N YAcad Sci 956:581-584

Chen Y, Huang WG, Zha DJ, Qiu JH, Wang JL, Sha SH, Schacht J (2007) Aspirin attenuates gentamicin ototoxicity: from the laboratory to the clinic. Hear Res 226:178-182

Chiang B, Fridman GY, Della Santina CC (2009) Enhancements to the Johns Hopkins multi-channel vestibular prosthesis yield reduced size, extended battery life, current steering and wireless control. Association for Research in Otolaryngology Abstract 867. Baltimore, MD

Cohen B, Suzuki J (1963a) Eye movements produced by vestibular nerve stimulation. Electroencephalogr Clin Neurophysiol 15:152

Cohen B, Suzuki JI (1963b) Eye movements induced by ampullary nerve stimulation. Am J Physiol 204:347

Cohen B, Suzuki JI (1964) Phases of central excitability evoked by ampullary nerve stimulation. Federation Proceedings 23:414

Cohen B, Shanzer S, Suzuki J (1963) Nystagmus induced by stimulation of single nerves from semicircular canals. Federation Proceedings 22:338 
Cohen B, Suzuki J, Bender MB (1964) Eye movements from semicircular canal nerve stimulation in cat. Ann Otol Rhinol Laryngol 73:153

CuRthoys IS, HaLmagy GM (1995) Vestibular compensation: a review of the oculomotor, neural, and clinical consequences of unilateral vestibular loss. J Vestib Res 5:67-107

Dai C, Fridman GY, Della Santina C (2010) Effects of vestibular electrode implantation and prosthetic stimulation on hearing in rhesus monkeys. Association for Research in Otolaryngology Annual Meeting

Davidovics N, Fridman GY, Della Santina CC (2009) Linearity of stimulus-response mapping during semicircular canal stimulation using a vestibular prosthesis. Association for Research in Otolaryngology Annual Meeting Abstract 339

Davidovics N, Fridman GY, Chiang B, Della Santina C (2010) Effects of stimulus pulse parameters on eye movement responses to stimulation delivered by a vestibular prosthesis. Association for Research in Otolaryngology Annual Meeting

Della Santina C, Migliaccio A, Patel A (2005a) Electrical stimulation to restore vestibular function development of a 3-D vestibular prosthesis. Conf Proc IEEE Eng Med Biol Soc 7:7380-7385

Della Santina C, Migliaccio A, Patel A, Carey JP, Park HJ, Anderson ICW, MiNOR L (2006) A multi-channel prosthesis for restoration of the 3-dimensional vestibulo-ocular reflex. Association for Research in Otolaryngology Abstract 298. Baltimore, MD

Della Santina CC, Migliaccio AA, Patel AH (2007) A multichannel semicircular canal neural prosthesis using electrical stimulation to restore 3-D vestibular sensation. IEEE Trans Biomed Eng 54:1016-1030

Della Santina CC, Potyagaylo V, Migliaccio Aa, Minor LB, Carey JP (2005b) Orientation of human semicircular canals measured by three-dimensional multiplanar CT reconstruction. JARO-Journal of the Association for Research in Otolaryngology 6:191-206

Della Santina CC, Migliaccio Aa, Park HJ, Anderson icW, Jiradejvong P, Minor LB, Carey JP (2005c) 3D Vestibuloocular reflex, afferent responses and crista histology in chinchillas after unilateral intratympanic gentamicin. Association for Research in Otolaryngology Annual Meeting. New Orleans

Diaz RC, LaRouere MJ, Bojrab DI, Zappia JJ, Sargent EW, Shaia WT (2007) Quality-of-life assessment of Meniere's disease patients after surgical labyrinthectomy. Otology \& Neurotology 28:74-86

Dickman JD, Beyer M, Hess BJ (2000) Three-dimensional organization of vestibular related eye movements to rotational motion in pigeons. Vision Res 40(20):2831-2844

EwaLd JR (1892) Physiologische untersuchugen uber das Endorgans des Nervus Octavus: Bergmann

Firszt JB, Holden LK, Skinner MW, Tobey EA, Peterson A, Gaggl W, Runge-SAmuelson CL, Wackym PA (2004) Recognition of speech presented at soft to loud levels by adult cochlear implant recipients of three cochlear implant systems. Ear Hear 25:375-387

Gillespie MB, Minor LB (1999) Prognosis in bilateral vestibular hypofunction. Laryngoscope 109:35-41

Goldberg JM (2000) Afferent diversity and the organization of central vestibular pathways. Exp Brain Res 130:277-297

Goldberg JM, Smith CE, Fernandez C (1984) Relation between discharge regularity and responses to externally applied galvanic currents in vestibular nerve afferents of the squirrel-monkey. J Neurophysiol 51:1236-1256

Gong WS, Merfeld DM (2000) Prototype neural semicircular canal prosthesis using patterned electrical stimulation. Ann Biomed Eng 28:572-581

Gong WS, Merfeld DM (2002) System design and performance of a unilateral horizontal semicircular canal prosthesis. IEEE Trans Biomed Eng 49:175-181

Gong WS, Haburcakova C, Merfeld DM (2008) Vestibulo-ocular responses evoked via bilateral electrical stimulation of the lateral semicircular canals. IEEE Trans Biomed Eng 55:2608-2619
Gorman PH, Mortimer JT (1983) The effect of stimulus parameters on the recruitment characteristics of direct nerve-stimulation. IEEE Trans Biomed Eng 30:407-414

Grunbauer WM, Dieterich M, Brandt T (1998) Bilateral vestibular failure impairs visual motion perception even with the head still. NeuroReport 9:1807-1810

Gstoettner WK, Adunka O, Franz P, Hamzavi J, Plenk H, Susani M, Baumgartner W, Kiefer J (2001) Perimodiolar electrodes in cochlear implant surgery. Acta Otolaryngol 121:216-219

HAY JC (1968) Visual adaptation to an altered correlation between eye movement and head movement. Science 160:429

Hendricks JL, Chikar JA, Crumling MA, Raphael Y, Martin DC (2008) Localized cell and drug delivery for auditory prostheses. Hear Res 242:117-131

Hess BJM, Angelaki DE (1997) Kinematic principles of primate rotational vestibulo-ocular reflex. 2. Gravity-dependent modulation of primary eye position. J Neurophysiol 78:2203-2216

Hirvonen TP, Minor LB, Hullar TE, Carey JP (2005) Effects of intratympanic gentamicin on vestibular afferents and hair cells in the chinchilla. J Neurophysiol 93:643-655

Hullar TE, Minor LB (1999) High-frequency dynamics of regularly discharging canal afferents provide a linear signal for angular vestibuloocular reflexes. J Neurophysiol 82:2000-2005

Hullar te, Della Santina CG, Hirvonen T, Lasker DM, Carey JP, MINOR LB (2005) Responses of irregularly discharging chinchilla semicircular canal vestibular-nerve afferents during high-frequency head rotations. J Neurophysiol 93:2777-2786

Jones GEG, Balaban CD, Jackson RL, WoOd KA, Kopke RD (2003) Effect of trans-bullar gentamicin treatment on guinea pig angular and linear vestibulo-ocular reflexes. Exp Brain Res 152:293-306

Lewis RF, Merfeld DM, Gong WS (2001) Cross-axis vestibular adaptation produced by patterned electrical stimulation. Neurology 56:A18-A18

Lewis RF, Haburcakova C, Merfeld DM (2008) Roll tilt psychophysics in rhesus monkeys during vestibular and visual stimulation. J Neurophysiol 100:140-153

Lewis RF, Haburcakova C, Gong W, Wall C, Merfeld DM (2009) Vestibular prosthesis tested in a vestibulopathic model. Association for Research in Otolaryngology. Baltimore, MD

Lewis RF, Gong WS, Ramsey M, Minor L, Boyle R, Merfeld DM (2002) Vestibular adaptation studied with a prosthetic semicircular canal. Journal of Vestibular Research-Equilibrium \& Orientation 12:87-94

Lyford-Pike S, Vogelheim C, Chu E, Della Santina CC, Carey JP (2007) Gentamicin is primarily localized in vestibular type I hair cells after intratympanic administration. JARO-Journal of the Association for Research in Otolaryngology 8:497-508

Ma CL, Billings P, Harris JP, Keithley EM (2000) Characterization of an experimentally induced inner ear immune response. Laryngoscope 110:451-456

Merfeld DM (2008) Sensor signal alignment. US Patent 7,454,246, November 18

Merfeld DM, Haburcakova C, Gong W, Lewis RF (2007) Chronic vestibulo-ocular reflexes evoked by a vestibular prosthesis. IEEE Trans Biomed Eng 54:1005-1015

Merfeld DM, Gong WS, Morrissey J, Saginaw M, Haburcakova C, LEWIS RF (2006) Acclimation to chronic constant-rate peripheral stimulation provided by a vestibular prosthesis. IEEE Trans Biomed Eng 53:2362-2372

Merrill DR, BiKson M, JeFFerYs JGR (2005) Electrical stimulation of excitable tissue: design of efficacious and safe protocols. J Neurosci Methods 141:171-198

Merwin WH JR, WALL C 3RD, Tomko DL (1989) The chinchilla's vestibulo-ocular reflex. Acta Otolaryngol 108:161-167

Middlebrooks JC, SNyder RL (2007) Auditory prosthesis with a penetrating nerve array. JARO-Journal of the Association for Research in Otolaryngology 8:258-279 
Migliaccio AA, MacDougall HG, Minor LB, Della Santina CC (2005) Inexpensive system for real-time 3-dimensional videooculography using a fluorescent marker array. J Neurosci Methods 143:141-150

Miles FA, Fuller JH (1974) Adaptive plasticity in vestibulo-ocular responses of rhesus-monkey. Brain Res 80:512-516

Miles FA, Fuller JH, Braitman DJ, Dow BM (1980) Long-term adaptive-changes in primate vestibuloocular reflex. 3. Electrophysiological observations in flocculus of normal monkeys. J Neurophysiol 43:1437-1476

Minor LB (1998) Gentamicin-induced bilateral vestibular hypofunction. JAMA-Journal of the American Medical Association 279:541-544

Robinson DA (1963) A method of measuring eye movement using a scleral search coil in a magnetic field. IEEE Trans Biomed Eng BM10:137

Robinson DA (1982) The use of matrices in analyzing the 3dimensional behavior of the vestibulo-ocular reflex. Biol Cybern 46:53-66

Robinson FP, MurPhy PG (1932) The validity of measuring eye movements by direct observation. Science 76:171-172

Ryugo DK, Kretzmer EA, Niparko JK (2005) Restoration of auditory nerve synapses in cats by cochlear implants. Science 310:14901492

SAdeghi SG, Minor LB, Cullen KE (2007a) Response of vestibularnerve afferents to active and passive rotations under normal conditions and after unilateral labyrinthectomy. J Neurophysiol 97:1503-1514

SAdeghi SG, Chacron MJ, Taylor MC, Cullen KE (2007b) Neural variability, detection thresholds, and information transmission in the vestibular system. J Neurosci 27:771-781

Scherer M, Migliaccio AA, Schubert MC (2008) Effect of vestibular rehabilitation on passive dynamic visual acuity. Journal of Vestibular Research-Equilibrium \& Orientation 18:147-157

Schubert MC, Della Santina CC, Shelhamer M (2008) Incremental angular vestibulo-ocular reflex adaptation to active head rotation. Exp Brain Res 191:435-446
Stakhovskaya O, Hradek GT, Snyder RL, Leake PA (2008) Effects of age at onset of deafness and electrical stimulation on the developing cochlear nucleus in cats. Hear Res 243:69-77

Suzuki J, Cohen B, Bender MB (1964) Compensatory eye movements induced by vertical semicircular canal stimulation. Exp Neurol 9:137

Suzuki JI, Cohen B (1964) Head eye body + limb movements from semicircular canal nerves. Exp Neurol 10:393

Suzuki JI, Goto K, Tokumasu K, Cohen B (1969) Implantation of electrodes near individual vestibular nerve branches in mammals. Ann Otol Rhinol Laryngol 78:815

Szturm T, Ireland DJ, Lessing-Turner M (1994) Comparison of different exercise programs in the rehabilitation of patients with chronic peripheral vestibular dysfunction. J Vestib Res 4:461479

UShIO M, LASker D, Minor L (2009) Unidirectional rotations in macaques produce asymmetric changes in gain of the horizontal VOR before and after unilateral labyrinthectomy (Abstract 760). Society for Neuroscience Annual Meeting 2009. Chicago, IL

Virrre E, Draper M, Gailey C, Miller D, Furness T (1998) Adaptation of the VOR in patients with low VOR gains. J Vestib Res 8:331-334

Wall C, Kos MI, GuYot JP (2007) Eye movements in response to electric stimulation of the human posterior ampullary nerve. Ann Otol Rhinol Laryngol 116:369-374

Weber KP, Aw ST, Todd MJ, McGarvie LA, Curthoys IS, Halmagy GM (2008) Head impulse test in unilateral vestibular loss: vestibulo-ocular reflex and catch-up saccades. Neurology 70:454-463

Weber KP, Aw ST, Todd MJ, McGarvie LA, Curthoys IS, Halmagy GM (2009) Horizontal head impulse test detects gentamicin vestibulotoxicity. Neurology 72:1417-1424

Wilson BS, DoRman MF (2008) Cochlear implants: a remarkable past and a brilliant future. Hear Res 242:3-21

Wilson VJ, Melvill J (1979) Mammalian vestibular physiology. Plenum, New York

Zeng FG (2004) Trends in cochlear implants. Trends in Amplification 8:1-34 